\title{
Shuttle Arylation by Rh(I) Catalyzed Reversible Carbon-Carbon Bond Activation of Unstrained Alcohols
}

\author{
Authors: \\ Marius D. R. Lutz ${ }^{1}$, Valentina C. M. Gasser ${ }^{1} \&$ Bill Morandi $^{1 *}$ \\ ${ }^{1}$ Laboratory of Organic Chemistry, Department of Chemistry and Applied Biosciences, ETH Zurich, Zurich, \\ Switzerland. \\ *Corresponding author. Email: bill.morandi@org.chem.ethz.ch
}

\begin{abstract}
The advent of transfer hydrogenation and borrowing hydrogen reactions paved the way to manipulate simple alcohols in previously unthinkable manners and circumvent the need for hydrogen gas. Analogously, transfer hydrocarbylation could greatly increase the versatility of tertiary alcohols. However, this reaction remains unexplored because of the challenges associated with the catalytic cleavage of unactivated $\mathrm{C}-\mathrm{C}$ bonds. Herein, we report a rhodium(I)-catalyzed shuttle arylation cleaving the $\mathrm{C}\left(\mathrm{sp}^{2}\right)-\mathrm{C}\left(\mathrm{sp}^{3}\right)$ bond in unstrained triaryl alcohols via a redoxneutral $\beta$-carbon elimination mechanism. A selective transfer hydrocarbylation of substituted (hetero)aryl groups from tertiary alcohols to ketones was realized, employing benign alcohols as latent $C$-nucleophiles. All preliminary mechanistic experiments support a reversible $\beta$-carbon elimination/migratory insertion mechanism. In a broader context, this novel reactivity offers a new platform for the manipulation of tertiary alcohols in catalysis.
\end{abstract}

\section{Main text}

Catalytic reversible reactions have found widespread use in the chemical community, as highlighted by the broad range of applications that alkene metathesis offers across the molecular sciences ${ }^{1-4}$. Another prominent example is the transfer hydrogenation reaction, in which primary and secondary alcohols and ketones are interconverted by formally shuttling a molecule of hydrogen, in lieu of using the hazardous gas (Fig. 1a, top) ${ }^{5}$. Many variants of this concept have been developed over the years, including borrowing hydrogen reactions that transiently dehydrogenate alcohols to construct new $\mathrm{C}-\mathrm{C}$ bonds ${ }^{6-11}$. The analogous transfer hydrocarbylation reaction using tertiary alcohols would provide exciting new avenues for the synthesis and modification of these species; however, reversibly cleaving and reforming a strong $\mathrm{C}-\mathrm{C}$ bond represents a far more considerable challenge (Fig. 1a, bottom).

Methods to directly activate $\mathrm{C}-\mathrm{C}$ bonds are attractive, as the substrates of interest can be functionalized without the need to introduce reactive functional handles, such as halides, and some have been strategically used in total synthesis ${ }^{12}$. When compared to the significant progress made in the functionalization of inert $\mathrm{C}-\mathrm{H}$ bonds, catalytic 
reactions to cleave strong $\mathrm{C}-\mathrm{C}$ bonds selectively are scarce ${ }^{13,14}$. Reasons for their low reactivity compared to $\mathrm{C}-\mathrm{H}$ bonds can be found in their kinetic inertness, as the $\sigma$-orbital is highly directional and does not allow for significant overlap with the d-orbitals of transition metals ${ }^{15,16}$.

Recently, several strategies to oxidatively cleave unstrained $\mathrm{C}-\mathrm{C}$ bonds using directing groups have been devised $^{14,15,17-20}$, however, there are few reports of unbiased systems that proceed via redox-neutral $\beta$-carbon elimination $^{16,18,21,22}$. While strategies to activate propargylic and allylic alcohols are well known, the activation of an unstrained $\mathrm{C}\left(\mathrm{sp}^{2}\right)-\mathrm{C}\left(\mathrm{sp}^{3}\right)$ bond remains challenging. We and others have reported cross-couplings of $\alpha, \alpha$-disubstituted arylmethanols with alkene electrophiles via directed $\mathrm{C}-\mathrm{C}$ bond activation forging new $\mathrm{C}-\mathrm{C}$ bonds ${ }^{23-28}$. Despite these, reports of selective $\mathrm{C}\left(\mathrm{sp}^{2}\right)-\mathrm{C}\left(\mathrm{sp}^{3}\right)$ bond cleavage of alcohols without using a directing group are rare and limited to specific substrate scaffolds designed to relieve internal strain or gain aromaticity ${ }^{29,30}$. A notable exception is a Pd-catalyzed biaryl coupling of $\alpha, \alpha$-disubstituted arylmethanols and aryl bromides reported by Miura ${ }^{31,32}$.

Continuing our interest in developing catalytic reversible reactions ${ }^{33-37}$, we sought to develop a shuttle catalysis reaction to interconvert tertiary alcohols and ketones via reversible $\mathrm{C}-\mathrm{C}$ bond cleavage (Fig. 1a, bottom). Such a reaction would parallel the widely employed transfer hydrogenation reaction between alcohols and ketones, with the key difference being that a $\mathrm{C}-\mathrm{C}$ bond would be cleaved and reformed instead of a $\mathrm{C}-\mathrm{H}$ bond.

Besides the inherent challenge of catalyzing the cleavage of a $\mathrm{C}-\mathrm{C}$ bond in a traditionally inert alcohol substrate (Fig. 1b, top), it is worth noting that there are only a few examples of transition metal-catalyzed aryl additions onto unactivated (i.e., aliphatic) ketones, even when employing traditional organoboron nucleophiles (Fig. 1b, bottom) ${ }^{38-}$ ${ }^{45}$. Reasons for the limited reports of insertions of ketones into late transition metal-carbon bonds include the high bond strength of the $\mathrm{C}=\mathrm{O} \pi$-bond, increased steric hinderance compared to terminal monosubstituted $\alpha$-olefins, and the less favorable interaction between a soft late transition metal center and an alkoxide ${ }^{46}$. Thus, the development of a shuttle catalysis approach to ketone arylation is contingent upon addressing two significant challenges in organometallic chemistry, namely the activation of unbiased $\mathrm{C}-\mathrm{C}$ bonds and the productive migratory insertion of a $\mathrm{M}-\mathrm{R}$ species into ketone substrates. A shuttle arylation protocol overcoming these challenges would open new research avenues that take advantage of reversible $\beta$-carbon elimination to parallel the ingenious reactions developed in the area of transfer hydrogenation and borrowing hydrogen reactions ${ }^{5-7,11}$. It would also pave the way for catalytic alternatives to highly reactive stoichiometric Grignard and organolithium additions. Crucial to unlocking the desired reactivity is the identification of a suitable catalyst that can lower the activation barrier of the key transition state for $\mathrm{C}-\mathrm{C}$ bond cleavage, which is identical to the one for $\mathrm{C}-\mathrm{C}$ bond formation by virtue of the microscopic reversibility principle (Fig. 1b, right).

Herein, we describe a reversible catalytic shuttle arylation reaction between triaryl alcohols and aliphatic ketones, by cleaving and reforming strong $\mathrm{C}-\mathrm{C}$ bonds (Fig. 1c). The reaction does not require directing groups or destabilization of the alcohol substrates and proceeds in the presence of a range of polar functional groups. Various aliphatic cyclic and linear ketones, including pharmaceuticals, could be arylated in good yields using this method. 


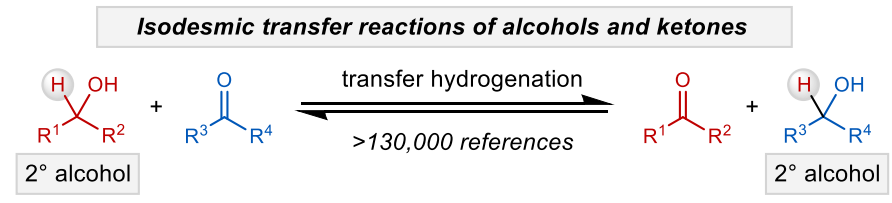

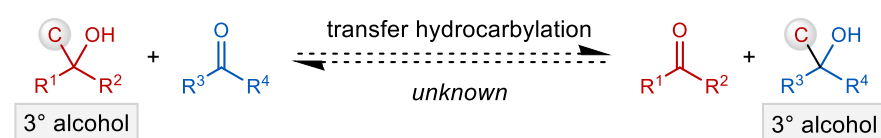

b

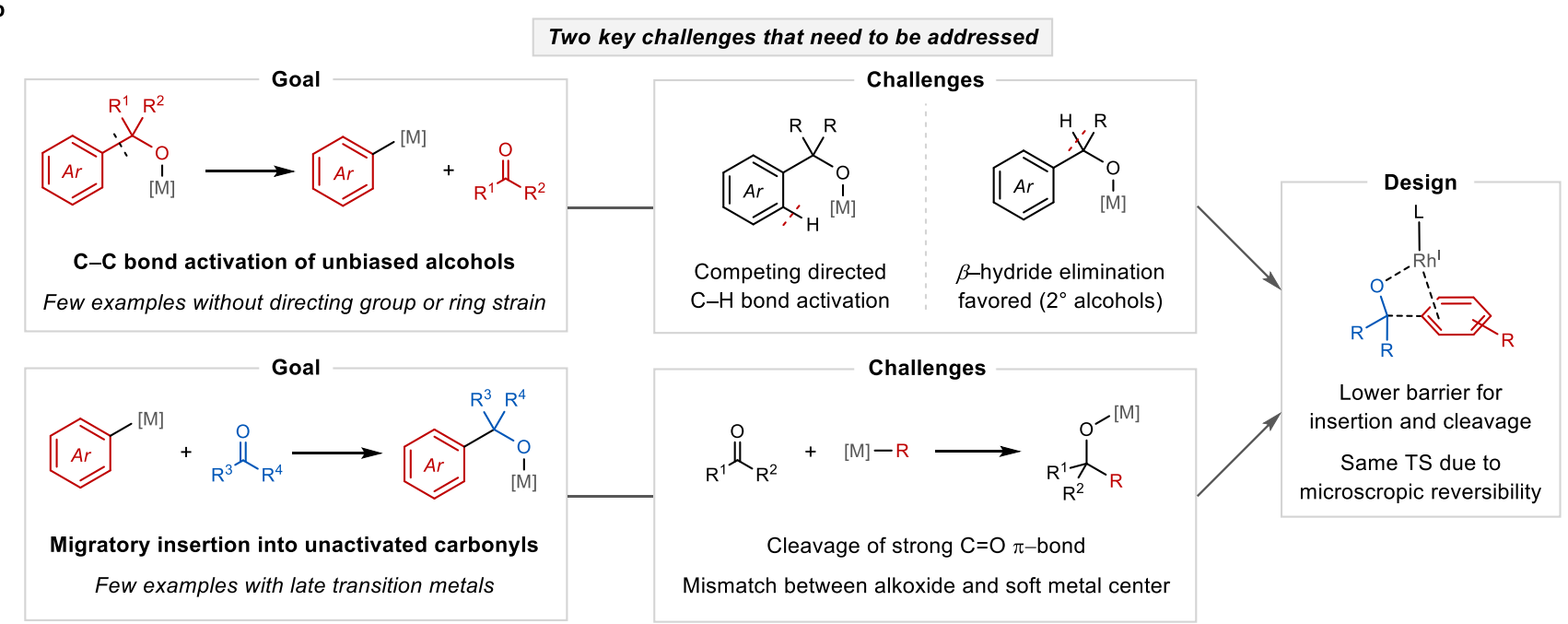

Reversible shuttle arylation of ketones enabled by C-C cleavage of alcohols (this work)

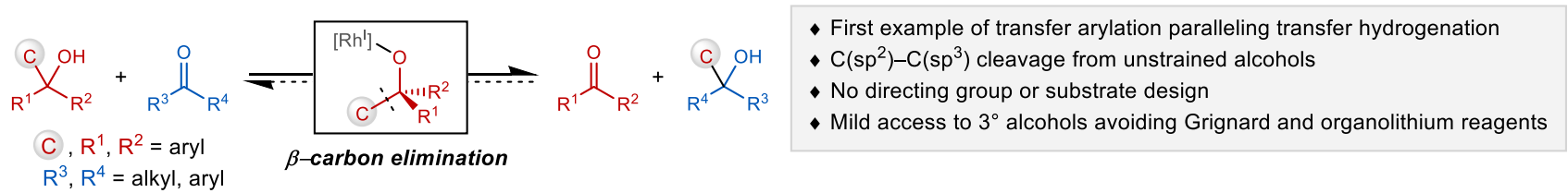

Fig. 1. Context of this work. a, Transfer hydrogenation is a widely employed reaction to interconvert $1^{\circ}$ and $2^{\circ}$ alcohols and carbonyl compounds. An analogous transfer hydrocarbylation of $3^{\circ}$ alcohols is unknown. $\mathbf{b}$, Two main challenges, namely activation of the $\mathrm{C}\left(\mathrm{sp}^{2}\right)-\mathrm{C}\left(\mathrm{sp}^{3}\right)$ bond in the alcohol and productive insertion into the ketone, have to be overcome to unlock the desired reactivity. Our strategy is to lower the barrier of the key transition state that occurs during both key steps. c, This work: Development of a catalytic shuttle arylation reaction between $3^{\circ}$ alcohols and ketones.

\section{Results}

Catalyst evaluation and reaction development. Inspired by stoichiometric $\beta$-carbon elimination studies from rhodium(I) alkoxide complexes by Hartwig and co-workers ${ }^{47-49}$, we envisaged that tuning the steric environment around the metal center to stabilize reactive, low coordinated complexes could unlock a catalytic transfer arylation tolerating a range of unbiased scaffolds. We began our studies with triphenylmethanol (1a) and 4,4'-difluorobenzophenone (2) as model substrates in the presence of $[\mathrm{Rh}(\operatorname{cod}) \mathrm{Cl}]_{2}$ and a weak base in toluene at $110{ }^{\circ} \mathrm{C}$. Initially, a panel of ligands was evaluated, and several conditions promoted benzophenone (3a) formation by $\beta$-carbon elimination but only trace amounts of alcohol product 4 were observed. After extensive reaction optimization, suitable conditions for the transfer of the phenyl group were identified (see Supplementary Information section 2 'Reaction 
development' for details). NHC ligands effected $\beta$-carbon elimination of 1a towards benzophenone (3a) and facilitated productive insertion of the aryl group into ketone 2. Among all NHC ligands tested, the electron-rich and sterically demanding ligand $\operatorname{IPr}^{*}{ }^{*}$ e was identified as the optimal ligand for this transformation (Fig. 2a) ${ }^{50}$. Interestingly, the major product was not triaryl alcohol $\mathbf{4}$, but diaryl ketone $\mathbf{5}$, which is formed through subsequent $\beta$ aryl elimination of the more electron-withdrawing group from the transiently generated alcohol $\mathbf{4}$ over the course of the reaction. After $48 \mathrm{~h}$ nearly full conversion of both alcohols 1a and $\mathbf{4}$ towards the ketones 3a and 5, and (fluoro)benzene was observed.

In an effort to avoid downstream reactions of the desired alcohol product, we next sought ways to take advantage of this unique reactivity to develop a synthetically attractive reaction. We reasoned that using an aliphatic ketone as acceptor would be beneficial because: 1 ) the tertiary alcohol product would likely not have the ability to scramble through cleavage of a strong $\mathrm{C}\left(\mathrm{sp}^{3}\right)-\mathrm{C}\left(\mathrm{sp}^{3}\right)$ bond, preventing undesired crossover reactions; 2$)$ the overall transfer reaction would likely be exothermic due to the formation of a conjugated ketone by-product as driving force (DFT predicts $\Delta \mathrm{G}=-4.0 \mathrm{kcal} \mathrm{mol}^{-1}$ ), while no deconjugation of the acceptor substrate (aliphatic ketone) would take place (see Supplementary Information section 7 'Computational studies' for details). Indeed, the reaction of 1a and 4,4dimethylcyclohexanone (6a) worked efficiently and reached full conversion with only marginal excess (1.5 equiv.) of the donor (Fig. 2b). The desired alcohol product 7a was obtained in excellent yield, while 1a was converted into ketone 3a and benzene as a side product (by protodemetalation). Control experiments confirmed the essential role of the catalyst and a weak base. Sterically encumbered NHC ligands resulted in the highest yield, but other ligands also effected product formation. Notably, the reaction could even be conducted at $0.5 \mathrm{~mol} \%$ catalyst loading, albeit with prolonged reaction time.

a

Proof of concept: reversible $\beta$-aryl elimination from triaryl alcohols

$\left[\mathrm{Rh}(\operatorname{cod}) \mathrm{Cl}_{2}(2.5 \mathrm{~mol} \%)\right.$

$\mathrm{IPr}^{*} \mathrm{OMe} \cdot \mathrm{HBF}_{4}(5 \mathrm{~mol} \%)$

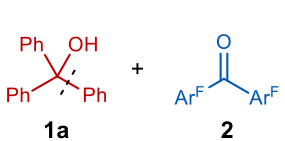

$\mathrm{KO} \mathrm{Bu}^{\mathrm{B}}(5 \mathrm{~mol} \%)$
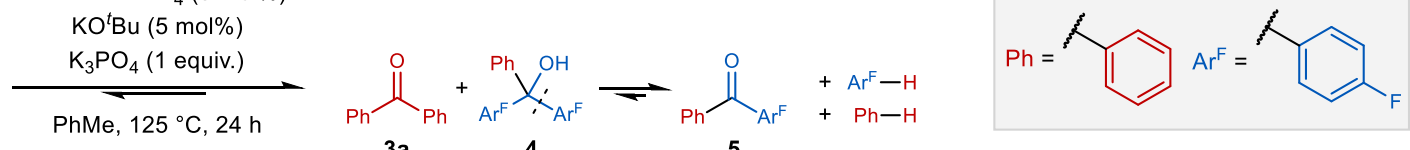

1 equiv.

2

aromatic ketone

PhMe, $125^{\circ} \mathrm{C}, 24 \mathrm{~h}$

3a

After $6 \mathrm{~h}: 19 \%$

After $24 \mathrm{~h}: 75 \%$

After $48 \mathrm{~h}: 83 \%$

scrambling of aryl groups - electron-deficient group is preferentially cleaved

more stable ketone products predominantly formed

4

$11 \%$
$7 \%$

$2 \%$

$3 \%$
$58 \%$
$86 \%$

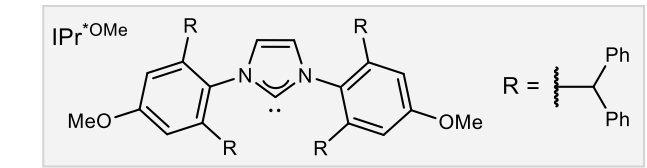

b

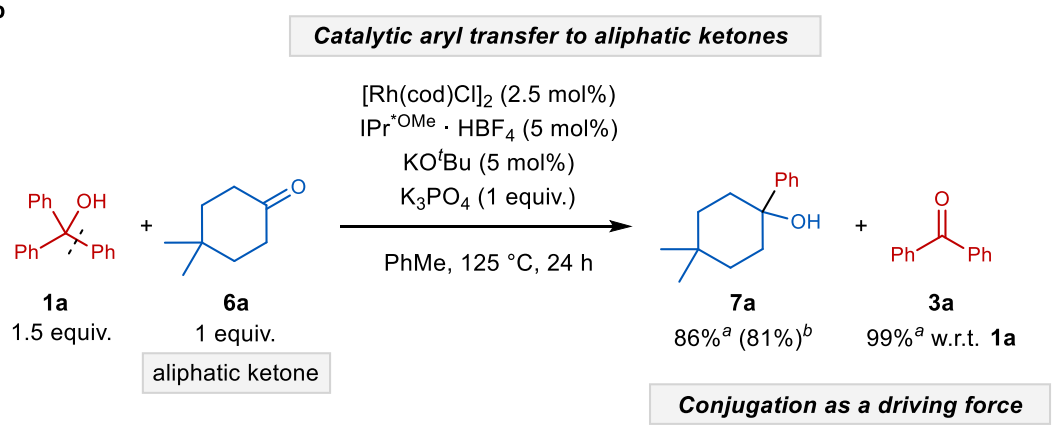

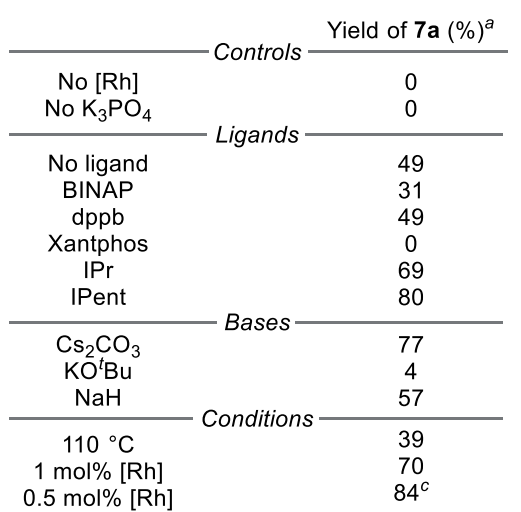


Fig. 2. Reaction development. a, Proof of concept of a catalytic reversible shuttle arylation reaction. Reaction conditions: 1a $(0.10 \mathrm{mmol}), 2(0.10 \mathrm{mmol}),[\mathrm{Rh}(\mathrm{cod}) \mathrm{Cl}]_{2}(2.5 \mathrm{~mol} \%), \mathrm{IPr}^{*} \mathrm{OMe} \cdot \mathrm{HBF}_{4}(5 \mathrm{~mol} \%), \mathrm{KO}^{t} \mathrm{Bu}(5 \mathrm{~mol} \%)$ and $\mathrm{K}_{3} \mathrm{PO}_{4}$ (1 equiv.) in toluene $(0.2 \mathrm{M})$ at $125^{\circ} \mathrm{C}$ for $24 \mathrm{~h}$. GC-FID yields using $n$-dodecane as an internal standard. b, Catalytic aryl transfer to aliphatic ketones. Reaction conditions: 1a $(0.15 \mathrm{mmol}), \mathbf{6 a}(0.10 \mathrm{mmol}),[\mathrm{Rh}(\mathrm{cod}) \mathrm{Cl}]_{2}$ (2.5 mol\%), IPr ${ }^{*} \mathrm{OMe} \cdot \mathrm{HBF}_{4}(5 \mathrm{~mol} \%), \mathrm{KO}^{t} \mathrm{Bu}(5 \mathrm{~mol} \%)$ and $\mathrm{K}_{3} \mathrm{PO}_{4}\left(1\right.$ equiv.) in toluene $(0.2 \mathrm{M})$ at $125{ }^{\circ} \mathrm{C}$ for $24 \mathrm{~h}$. ${ }^{a}$ GC-FID yields using $n$-dodecane as an internal standard. ${ }^{b}$ Isolated yield after purification. ${ }^{c} 72$ hours.

Substrate scope. After finding the optimal conditions, we investigated the generality and utility of this transformation. A range of cyclic (6a-b) and linear (6d-e) aliphatic ketones were efficiently arylated to afford the corresponding tertiary alcohols in good yields (Fig. 3a). Acetophenone could also be arylated, albeit the formation of product $7 \mathbf{c}$ was reversible, leading to a diminished yield. Several functional groups were found to be compatible with the reaction conditions, such as acetal-protected ketones (7f), protected amines $(\mathbf{7 g})$, ethers (7h), and sulfones (7i). Notably, both carboxylic esters (7k-l) and amides (7m) remained untouched. Cross-coupling handles such as aryl silanes $(\mathbf{7 j})$ and chlorides $(\mathbf{7 n})$ remained unchanged, offering the potential for orthogonal synthetic manipulation. The arylation also proved to be effective in the presence of a heterocyclic residue (7o). Drug molecules, such as nabumetone and pentoxifylline, underwent arylation in good yields (7p-q), demonstrating the potential for latestage derivatization of bioactive compounds. Sterically hindered ketones were unreactive under the reaction conditions, which might indicate that bulky ketones are unable to approach the sterically encumbered catalyst (Supplementary Table S12).

Subsequently, we turned our focus to the alcohol scope. We first investigated several symmetrical triaryl alcohols 1 (Fig. 3b). A range of alkyl-substituted alcohols (8a-c) and a 2-naphthyl group (8d) were suitable donor molecules, affording the desired alcohols in good to excellent yield. Moreover, several common functional groups were tolerated, such as fluoro $(\mathbf{8 e})$, chloro $(\mathbf{8 f})$, methoxy $(\mathbf{8 g})$, trifluoromethyl $(\mathbf{8 h}, \mathbf{8 j})$, and trifluoromethoxy $(\mathbf{8 i})$ groups. The reaction was efficient with both electron-rich and -deficient donors, albeit the yield of $\mathbf{8 g}$ and $\mathbf{8 j}$ was limited due to fast consumption of the alcohol starting material via protodemetalation. Notably, heterocyclic scaffolds were well tolerated under the reaction conditions and allowed the transfer of 1,3-benzodioxolane $(\mathbf{8 k})$, benzofurane $(\mathbf{8 1}$-m), and morpholine (8n) moieties.

Beyond triaryl alcohols, we investigated the propensity of other alcohols to undergo $\beta$-carbon elimination (Fig. 3c). 1,1-Diphenylethanol (1p) afforded product 9a in moderate yield, because the formation of the by-product acetophenone is reversible (7c, vide supra). Excitingly, a $2^{\circ}$ alcohol (1q) could be engaged to afford the product $\mathbf{9 b}$ in a promising yield, showing potential for productive $\beta$-carbon elimination in the presence of a competing $\beta$-hydride. We surmise that rapid and reversible $\beta$-hydride elimination occurs under the reaction conditions, yet the final formation of the desired tertiary alcohol kinetically traps the occasionally formed $\beta$-carbon elimination intermediate, hence slowly driving the mixture towards the desired product. This result thus suggests the possibility to merge complex sequences of reversible $\beta$-hydride and $\beta$-carbon eliminations, opening new avenues for the manipulation of alcohols in catalysis.

To further showcase the scalability and robustness of this reaction, the transfer arylation of $\mathbf{1 a}$ and $\mathbf{6 d}$ was conducted on $10 \mathrm{mmol}$ scale with a reduced catalyst loading of $1 \mathrm{~mol} \%$ (Fig. $3 \mathrm{~d}$ ). The alcohol product $\mathbf{7 d}$ ' was obtained in 
$70 \%$ yield after purification. The second product, benzophenone (3a'), was recovered in $77 \%$ with respect to $1 \mathbf{a}$, or $116 \%$ based on the limiting reagent $\mathbf{6 d}$. 


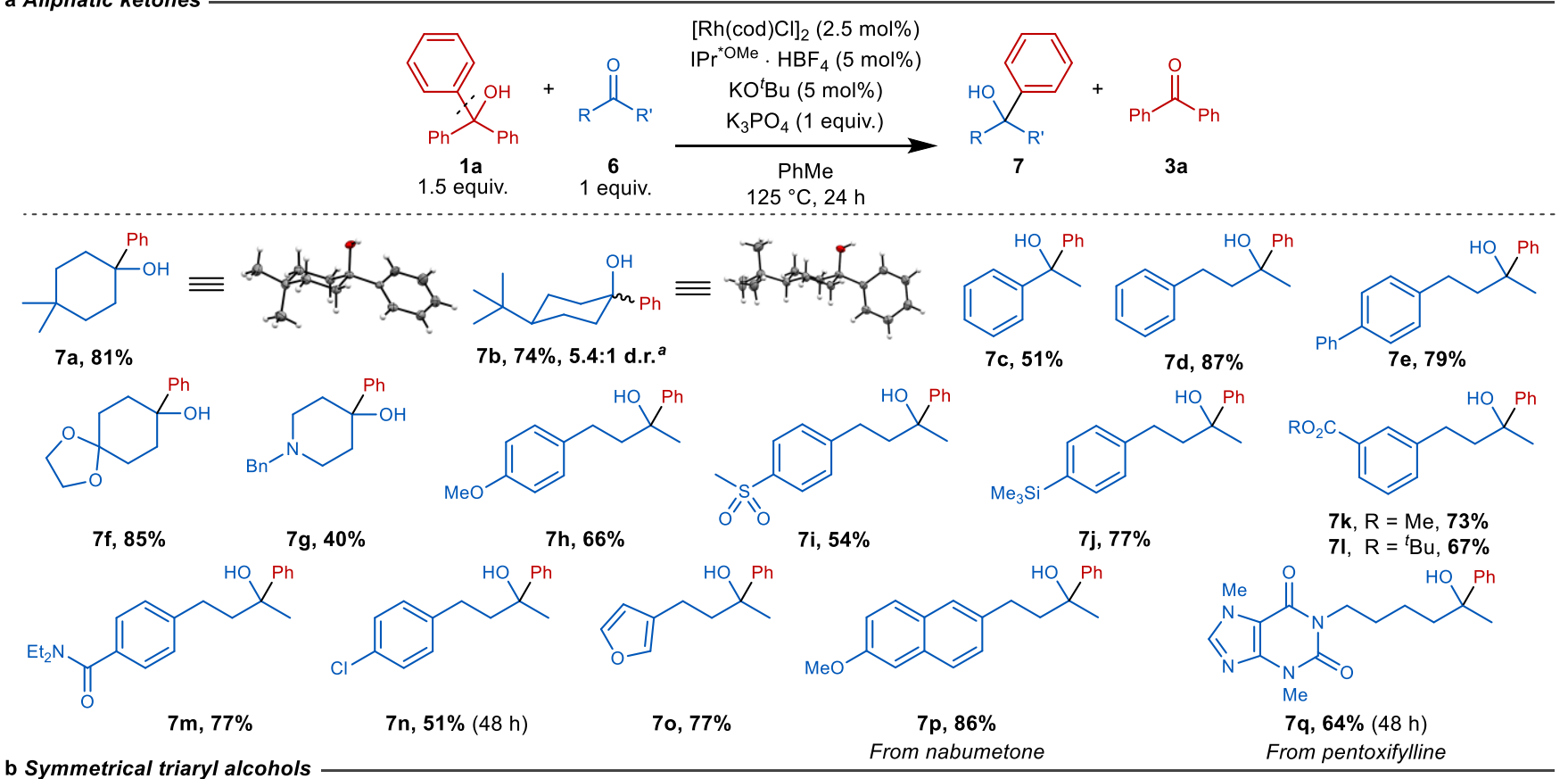

b Symmetrical triaryl alcohols
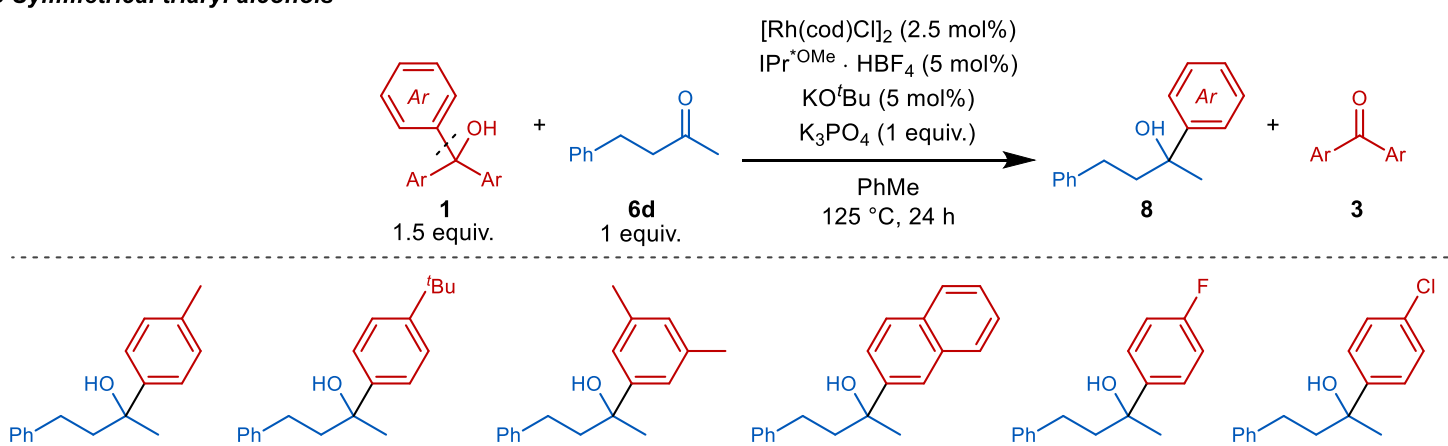

$8 a, 68 \%$

$8 b, 51 \%$

$8 c, 70 \%$

$8 d, 87 \%$

$8 \mathrm{e}, 68 \%$

$8 f, 76 \%$

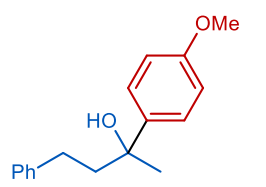<smiles>CC(O)(CCc1ccccc1)c1ccc(C(C)(O)CCc2ccccc2)cc1</smiles>

$8 \mathrm{~h}, 80 \%$

$8 \mathrm{i}, 75 \%$
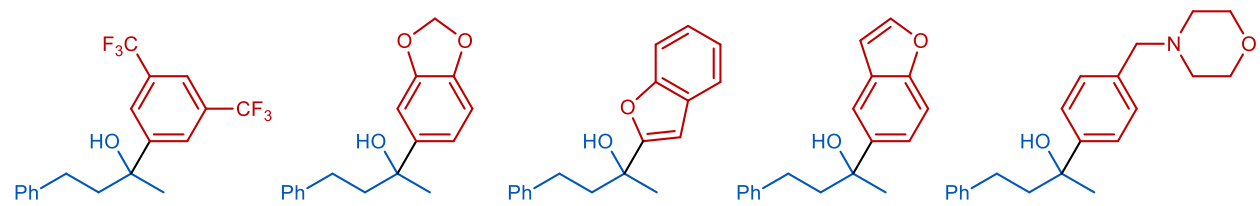

c Non-symmetrical alcohols

$8 \mathrm{j}, 39 \%$

$8 \mathrm{k}, 46 \%$

$81,68 \%$

$8 \mathrm{~m}, 55 \%$

$8 \mathrm{n}, 69 \%$

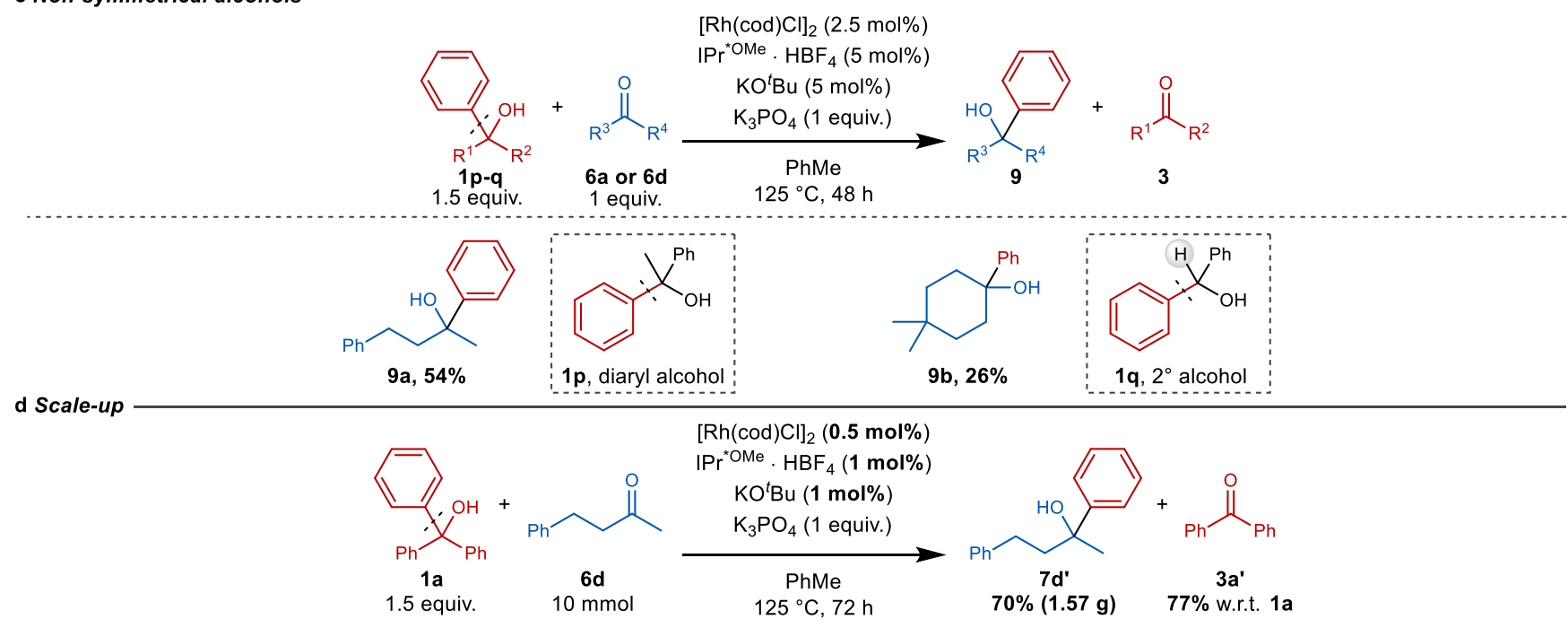


Fig. 3. Substrate scope of the shuttle arylation. a, Scope of ketones. b, Scope of symmetric alcohols. c, Scope of asymmetric alcohols. d, Scale-up at reduced catalyst loading. ${ }^{a}$ Ratio determined by ${ }^{1} \mathrm{H}$ NMR of the crude reaction mixture.

Preliminary mechanistic studies. After demonstrating the synthetic versatility of this protocol, we performed experiments to investigate the reaction's reversibility. To confirm that $\beta$-carbon elimination is reversible for triaryl alcohols 1, diaryl ketone 3b was added to the reaction mixture of $\mathbf{1 a}$ and $\mathbf{6 a}$ (Fig. 4a). Incorporation of both the phenyl group (red) and the $p$-tolyl group (blue) into 6a was observed in a $4.9: 1$ ratio, together with a mixture of all three possible diaryl ketones, confirming that addition into diaryl ketone $\mathbf{3 b}$ is reversible and has a similar rate to the addition into aliphatic ketone $\mathbf{6 a}$.

To evaluate the reversibility of the reaction in the case of (dialkyl)aryl alcohols 7, alcohol trans-7b and benzophenone (3a) were subjected to the reaction conditions (Fig. 4b). Notably, isomerization to the more stable cis-isomer and small amounts of ketone $\mathbf{6 b}$ were observed, highlighting that $\beta$-aryl elimination from (dialkyl)aryl alcohols also takes place, albeit the reaction rate is much lower than for triaryl alcohols.

To gain insight into the electronic preference of $\beta$-aryl elimination, intermolecular competition experiments with two symmetrical triaryl alcohols were carried out (Fig. 4c). Interestingly, both electron-rich and -deficient substituted aryl groups were preferentially cleaved compared to phenyl, with no correlation between the electronic character and the observed selectivity. Notably, a similar trend was observed in Pd-catalyzed $\beta$-aryl elimination ${ }^{51}$. The interpretation of these results is, however, complicated by the partial reversibility of the addition of one aryl group to another diaryl ketone by-product leading to crossover products (see Fig. 4a).

We next independently synthesized the complex $\left[\mathrm{Rh}\left(\mathrm{IPr}^{*} \mathrm{OMe}\right)(\mathrm{cod}) \mathrm{Cl}\right](\mathbf{1 0})$ that is presumedly formed in situ (Fig. 4d). The complex was catalytically competent, effectively giving the same yield of product (Supplementary Table S6). The crystal structure obtained by X-ray diffraction of the air-stable complex illustrates the steric encumbrance of the NHC ligand around the substrate binding site. The steric influence of the ligand was parameterized by its buried volume $\left(V_{\text {bur }}\right)^{52}$. While the buried volume of $\operatorname{IPr}^{*}$ OMe is slightly larger than that of IPr at $3.5 \AA$ radius $\left(V_{\text {bur }}=\right.$ $37.2 \%$ versus $33.6 \%$ ), its reach extends further in the periphery ( $V_{\text {bur }}=46.4 \%$ versus $38.6 \%$ at $5.5 \AA$ ).

Based on these preliminary observations, we propose the following reversible catalytic cycle (Fig. 4e). Ligand exchange of complex I (10) with the alcohol substrate followed by dissociation of a cod ligand creates the presumed catalytically active species II. As in the case of $\beta$-hydride elimination, an empty coordination site is a requisite for $\beta$-carbon elimination to occur. The coordinatively unsaturated Rh center in II likely interacts with one of the aryl rings in a $\eta^{2}$-fashion, as has been shown in X-ray structures of related complexes ${ }^{47}$, thereby facilitating the $\mathrm{C}-\mathrm{C}$ bond cleavage. Aryl complex III then undergoes reversible ketone exchange to form complex IV that in turn undergoes migratory insertion into the ketone to form alkoxide complex $\mathbf{V}$. Every step in the catalytic cycle is reversible according to our findings. Finally, alcohol exchange closes the cycle. 


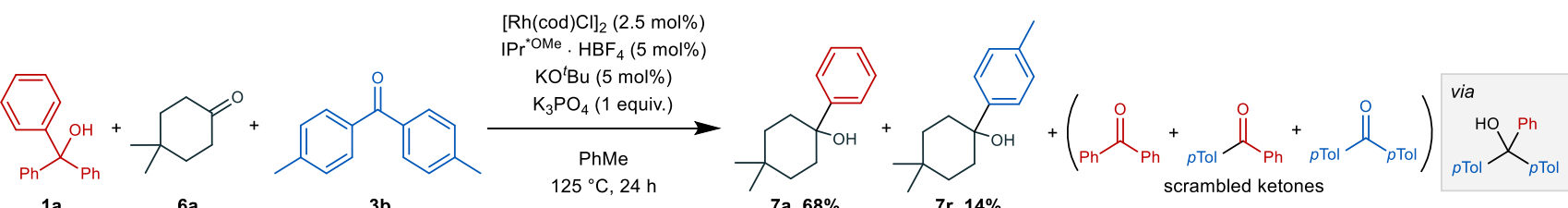
$\begin{array}{cc}\mathbf{1 a} & \mathbf{6 a} \\ 1.5 \text { equiv. } & 1.0 \text { equiv. }\end{array}$

1.5 equiv.

$125{ }^{\circ} \mathrm{C}, 24 \mathrm{~h} 7 \mathrm{7a}, 68 \%$

$7 r, 14 \%$

scrambled ketones

Reversible addition into diaryl ketone leads to two alcohol products

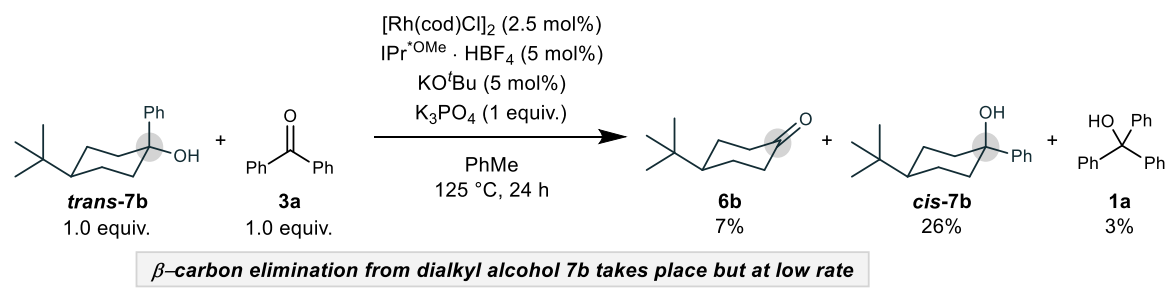

c

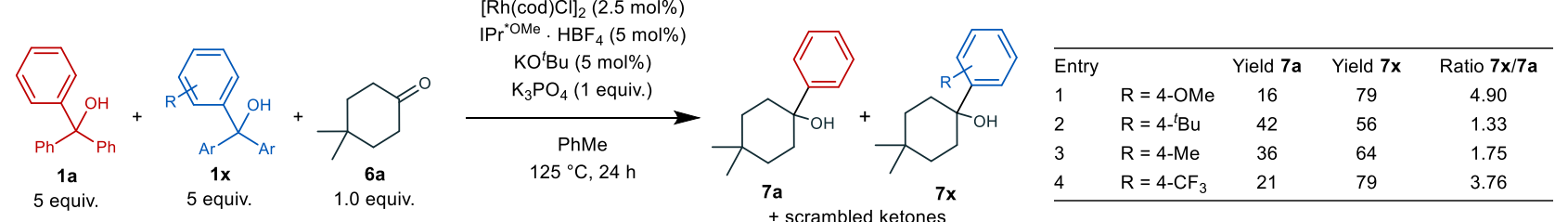

no correlation with electronic character
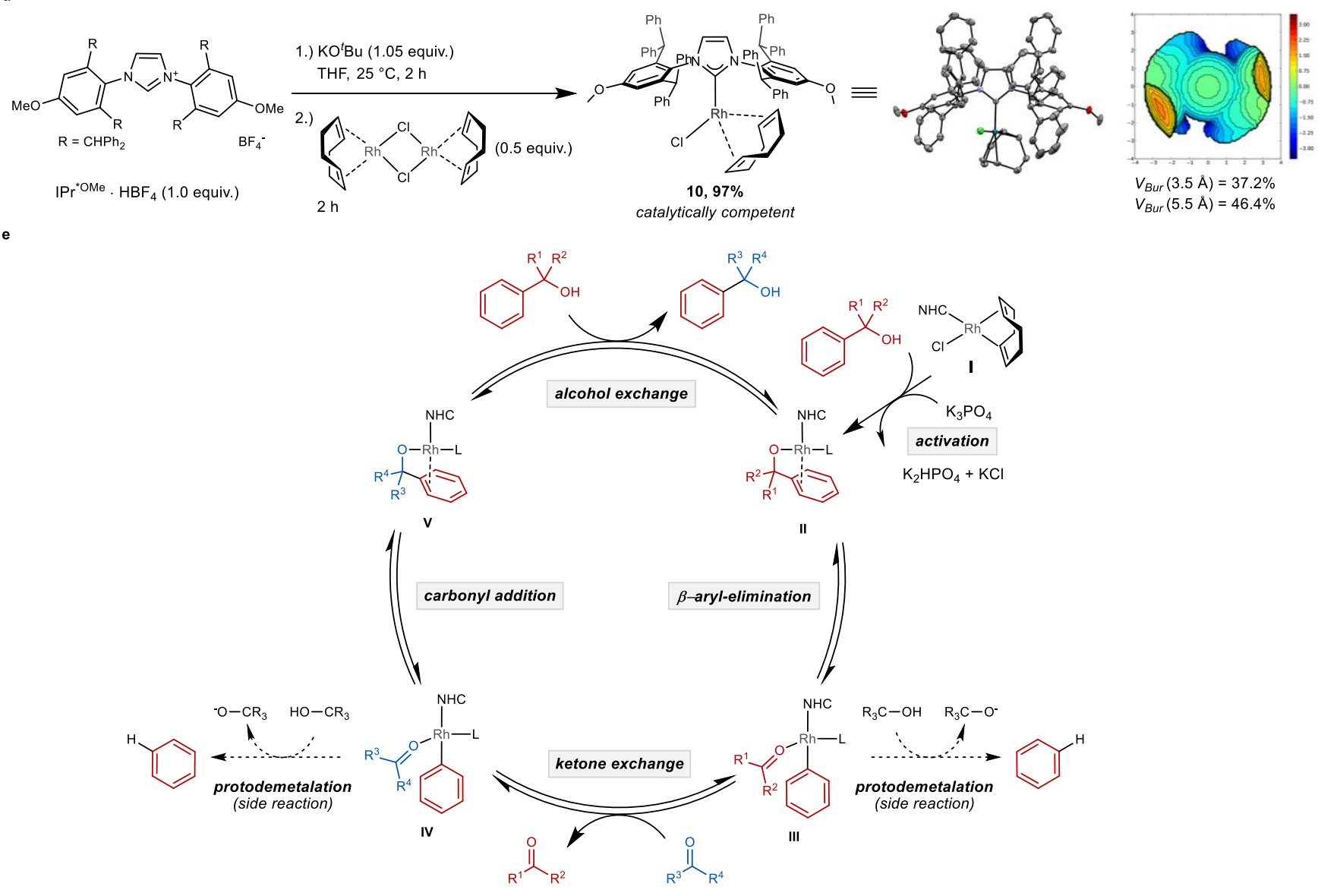

Fig. 4. Preliminary mechanistic experiments and proposed catalytic cycle. a, $\beta$-Aryl elimination from triaryl alcohols is reversible, resulting in two alcohol products via scrambling. $\mathbf{b}, \beta$-Aryl elimination from di(alkyl)aryl alcohols is feasible but occurs at a low rate. c, Intermolecular competition experiments show no correlation between the electronic character of the cleaved aryl group and product selectivity. $\mathbf{d}$, The catalytically active complex $\mathbf{1 0}$ was independently synthesized and characterized by X-ray crystallography. The NHC ligand displays a large buried volume in the periphery. e, Proposed catalytic cycle. 


\section{Conclusions}

In summary, we have developed a catalytic shuttle arylation reaction that cleaves unactivated $\mathrm{C}\left(\mathrm{sp}^{2}\right)-\mathrm{C}\left(\mathrm{sp}^{3}\right)$ bonds in unstrained alcohols and arylates unactivated aliphatic ketones. This is the first example of a reversible transfer hydrocarbylation reaction that parallels the ubiquitous transfer hydrogenation reaction of secondary alcohols and carbonyl acceptors. Using this method, triaryl alcohols can be used as latent nucleophilic aryl sources for traditionally challenging catalytic additions to ketones. Preliminary mechanistic studies point towards reversible $\mathrm{C}-\mathrm{C}$ bond activation as a key factor in enabling this process. In a broader context, this novel reactivity offers numerous opportunities for the creative use of tertiary alcohols in synthesis which parallel major achievements made in transfer hydrogenation and borrowing hydrogen.

\section{Data availability}

Materials and methods, experimental procedures, detailed optimization studies, mechanistic studies and NMR spectra are available in the Supplementary Information or from the corresponding author upon reasonable request. Crystallographic data for compounds $\mathbf{7 a}$, cis-7b and $\mathbf{1 0}$ are available free of charge from the Cambridge Crystallographic Data Centre under deposition numbers 2035749, 2035750 and 2035751. Copies of the data can be obtained free of charge via https://www.ccdc.cam.ac.uk/structures/.

\section{References}

1. Grubbs, R. H. Handbook of Metathesis. (Wiley-VCH, 2003).

2. $\quad$ Fürstner, A. Olefin Metathesis and Beyond. Angew. Chem. Int. Ed. 39, 3012-3043 (2000).

3. Schrock, R. R. \& Hoveyda, A. H. Molybdenum and tungsten imido alkylidene complexes as efficient olefinmetathesis catalysts. Angew. Chem. Int. Ed. 42, 4592-4633 (2003).

4. Ogba, O. M., Warner, N. C., O’Leary, D. J. \& Grubbs, R. H. Recent advances in ruthenium-based olefin metathesis. Chem. Soc. Rev. 47, 4510-4544 (2018).

5. Wang, D. \& Astruc, D. The Golden Age of Transfer Hydrogenation. Chem. Rev. 115, 6621-6686 (2015).

6. Hamid, M. H. S. A., Slatford, P. A. \& Williams, J. M. J. Borrowing hydrogen in the activation of alcohols. Adv. Synth. Catal. 349, 1555-1575 (2007).

7. Corma, A., Navas, J. \& Sabater, M. J. Advances in One-Pot Synthesis through Borrowing Hydrogen Catalysis. Chem. Rev. 118, 1410-1459 (2018).

8. Dobereiner, G. E. \& Crabtree, R. H. Dehydrogenation as a substrate-activating strategy in homogeneous transition-metal catalysis. Chem. Rev. 110, 681-703 (2010).

9. Zbieg, J. R., Yamaguchi, E., McInturff, E. L. \& Krische, M. J. Enantioselective C-H crotylation of primary alcohols via hydrohydroxyalkylation of butadiene. Science 336, 324-327 (2012).

10. Goldman, A. S. et al. Catalytic alkane metathesis by tandem alkane dehydrogenation-olefin metathesis. Science 312, 257-261 (2006).

11. Gunanathan, C. \& Milstein, D. Applications of acceptorless dehydrogenation and related transformations in chemical synthesis. Science 341, 257-261 (2013).

12. Wang, B., Perea, M. A. \& Sarpong, R. Transition-Metal-Mediated Cleavage of C - C Single Bonds: Making the Cut in Total Synthesis. Angew. Chem. Int. Ed. 59, 2-24 (2020).

13. Miura, M. \& Satoh, T. Catalytic Processes Involving $\beta$-Carbon Elimination. Palladium in Organic Synthesis. Topics in Organometallic Chemistry (Springer, 2005).

14. Dong, G. C-C Bond Activation. (Springer, 2014).

15. Rybtchinski, B. \& Milstein, D. Metal Insertion into C-C Bonds in Solution. Angew. Chem. Int. Ed. 38, 870883 (1999).

16. Lutz, M. D. R. \& Morandi, B. Metal-Catalyzed Carbon-Carbon Bond Cleavage of Unstrained Alcohols. 
Chem. Rev. http://dx.doi.org/10.1021/acs.chemrev.0c00154 (2020).

17. Jun, C.-H. Transition metal-catalyzed carbon-carbon bond activation. Chem. Soc. Rev. 33, 610-618 (2004).

18. Chen, F., Wang, T. \& Jiao, N. Recent Advances in Transition-Metal-Catalyzed Functionalization of Unstrained Carbon-Carbon Bonds. Chem. Rev. 114, 8613-8661 (2014).

19. Souillart, L. \& Cramer, N. Catalytic C-C Bond Activations via Oxidative Addition to Transition Metals. Chem. Rev. 115, 9410-9464 (2015).

20. Xia, Y. \& Dong, G. Temporary or removable directing groups enable activation of unstrained $\mathrm{C}-\mathrm{C}$ bonds. Nature Reviews Chemistry 1-15 (2020).

21. Murakami, M. Cleavage of Carbon-Carbon Single Bonds by Transition Metals. (Wiley-VCH, 2015).

22. Song, F., Gou, T., Wang, B.-Q. \& Shi, Z.-J. Catalytic activations of unstrained C-C bond involving organometallic intermediates. Chem. Soc. Rev. 47, 7078-7115 (2018).

23. $\mathrm{Li}, \mathrm{H}$. et al. Pyridinyl directed alkenylation with olefins via $\mathrm{Rh}(\mathrm{III})$-catalyzed $\mathrm{C}-\mathrm{C}$ bond cleavage of secondary arylmethanols. J. Am. Chem. Soc. 133, 15244-15247 (2011).

24. Chen, K. et al. Direct oxidative arylation via rhodium-catalyzed C-C bond cleavage of secondary alcohols with arylsilanes. Chem. Sci. 3, 1645-1649 (2012).

25. Zhang, X.-S. et al. Rh-Catalyzed C-C Cleavage of Benzyl/Allylic Alcohols to Produce Benzyl/Allylic Amines or other Alcohols by Nucleophilic Addition of Intermediate Rhodacycles to Aldehydes and Imines. Chem. Eur. J. 18, 16214-16225 (2012).

26. Ozkal, E., Cacherat, B. \& Morandi, B. Cobalt(III)-Catalyzed Functionalization of Unstrained CarbonCarbon Bonds through $\beta$-Carbon Cleavage of Alcohols. ACS Catal. 5, 6458-6462 (2015).

27. Wang, H., Choi, I., Rogge, T., Kaplaneris, N. \& Ackermann, L. Versatile and robust C-C activation by chelation-assisted manganese catalysis. Nat. Catal. 1, 993-1001 (2018).

28. Qiu, Y., Scheremetjew, A. \& Ackermann, L. Electro-Oxidative C-C Alkenylation by Rhodium(III) Catalysis. J. Am. Chem. Soc. 141, 2731-2738 (2019).

29. Nishimura, T., Katoh, T. \& Hayashi, T. Rhodium-catalyzed aryl transfer from trisubstituted aryl methanols to $\alpha, \beta$-unsaturated carbonyl compounds. Angew. Chem. Int. Ed. 46, 4937-4939 (2007).

30. Deng, R., Xi, J., Li, Q. \& Gu, Z. Enantioselective Carbon-Carbon Bond Cleavage for Biaryl Atropisomers Synthesis. Chem 5, 1834-1846 (2019).

31. Terao, Y., Wakui, H., Satoh, T., Miura, M. \& Nomura, M. Palladium-Catalyzed Arylative Carbon-Carbon Bond Cleavage of $\alpha, \alpha$-Disubstituted Arylmethanols. J. Am. Chem. Soc. 123, 10407-10408 (2001).

32. Terao, Y. et al. Palladium-catalyzed arylation of $\alpha, \alpha$-disubstituted arylmethanols via cleavage of a $\mathrm{C}-\mathrm{C}$ or a C-H bond to give biaryls. J. Org. Chem. 68, 5236-5243 (2003).

33. Bhawal, B. N. \& Morandi, B. Catalytic transfer functionalization through shuttle catalysis. ACS Catal. 6, $7528-7535$ (2016).

34. Lian, Z., Bhawal, B. N., Yu, P. \& Morandi, B. Palladium-catalyzed carbon-sulfur or carbon-phosphorus bond metathesis by reversible arylation. Science 356, 1059-1063 (2017).

35. Xianjie, F., Yu, P. \& Morandi, B. Catalytic reversible alkene-nitrile interconversion through controllable transfer hydrocyanation. Science 351, 832-836 (2016).

36. Bhawal, B. N. \& Morandi, B. Catalytic Isofunctional Reactions-Expanding the Repertoire of Shuttle and Metathesis Reactions. Angew. Chem. Int. Ed. 58, 10074-10103 (2019).

37. Bhawal, B. N., Reisenbauer, J. C., Ehinger, C. \& Morandi, B. Overcoming Selectivity Issues in Reversible Catalysis: A Transfer Hydrocyanation Exhibiting High Kinetic Control. J. Am. Chem. Soc. 142, 1091410920 (2020).

38. Liu, Y.-L. \& Lin, X.-T. Recent Advances in Catalytic Asymmetric Synthesis of Tertiary Alcohols via Nucleophilic Addition to Ketones. Adv. Synth. Catal. 361, 876-918 (2019).

39. Ueura, K., Miyamura, S., Satoh, T. \& Miura, M. Rhodium-catalyzed addition of arylboron compounds to nitriles, ketones, and imines. J. Organomet. Chem. 691, 2821-2826 (2006).

40. Liao, Y.-X., Xing, C.-H. \& Hu, Q.-S. Rhodium(I)/Diene-Catalyzed Addition Reactions of Arylborons with Ketones. Org. Lett. 14, 1544-1547 (2012).

41. White, J. R., Price, G. J., Plucinski, P. K. \& Frost, C. G. The rhodium-catalysed 1,2-addition of arylboronic acids to aldehydes and ketones with sulfonated S-Phos. Tetrahedron Lett. 50, 7365-7368 (2009).

42. Huang, L. et al. Highly Enantioselective Rhodium-Catalyzed Addition of Arylboroxines to Simple Aryl Ketones: Efficient Synthesis of Escitalopram. Angew. Chem. Int. Ed. 55, 4527-4531 (2016).

43. Gallego, G. M. \& Sarpong, R. Rh(I)-catalyzed enantioselective intramolecular hydroarylation of unactivated ketones with aryl pinacolboronic esters. Chem. Sci. 3, 1338-1342 (2012).

44. Korenaga, T., Ko, A., Uotani, K., Tanaka, Y. \& Sakai, T. Synthesis and application of 2,6- 
bis(trifluoromethyl)-4-pyridyl phosphanes: The most electron-poor aryl phosphanes with moderate bulkiness. Angew. Chem. Int. Ed. 50, 10703-10707 (2011).

45. Hatano, M. \& Ishihara, K. Recent progress in the catalytic synthesis of tertiary alcohols from ketones with organometallic reagents. Synth. 1647-1675 (2008).

46. Krug, C. \& Hartwig, J. F. Reactions of an arylrhodium complex with aldehydes, imines, ketones, and alkynones. New classes of insertion reactions. Organometallics 23, 4594-4607 (2004).

47. Zhao, P., Incarvito, C. D. \& Hartwig, J. F. Direct observation of $\beta$-aryl eliminations from Rh(I) alkoxides. J. Am. Chem. Soc. 128, 3124-3125 (2006).

48. Zhao, P., Incarvito, C. D. \& Hartwig, J. F. Carbon-oxygen bond formation between a terminal alkoxo ligand and a coordinated olefin. Evidence for olefin insertion into a rhodium alkoxide. J. Am. Chem. Soc. 128, 9642-9643 (2006).

49. Zhao, P. \& Hartwig, J. F. Insertions of ketones and nitriles into organorhodium(i) complexes and $\beta$ hydrocarbyl eliminations from rhodium(I) alkoxo and iminyl complexes. Organometallics 27, 4749-4757 (2008).

50. Meiries, S., Speck, K., Cordes, D. B., Slawin, A. M. Z. \& Nolan, S. P. [Pd(IPr*OMe)(acac)Cl]: Tuning the N-Heterocyclic Carbene in Catalytic C-N Bond Formation. Organometallics 32, 330-339 (2013).

51. Bour, J. R., Green, J. C., Winton, V. J. \& Johnson, J. B. Steric and electronic effects influencing $\beta$-aryl elimination in the Pd-catalyzed carbon-carbon single bond activation of triarylmethanols. J. Org. Chem. 78, 1665-1669 (2013).

52. Clavier, H. \& Nolan, S. P. Percent buried volume for phosphine and N-heterocyclic carbene ligands: steric properties in organometallic chemistry. Chem. Commun. 46, 841 (2010).

\section{Acknowledgements}

We thank the European Research Council (ERC Grant 757608 ShuttleCat), the Swiss National Science Foundation (SNF 184658) and the ETH Zürich for financial support. M.D.R.L. is grateful for funding from the German Academic Scholarship Foundation. We thank the NMR service, the Molecular and Biomolecular Analysis Service (MoBiAS) and X-ray departments of ETH Zürich for technical assistance. We thank the Morandi group members for critical proof reading of the manuscript.

\section{Author contributions}

M.L. and B.M. conceived the project. M.L. discovered and developed the reaction; M.L. and V.G. conducted the synthetic studies. All authors contributed to the writing and editing of the manuscript.

\section{Competing interests}

The authors declare no competing interests. 